\title{
Rituximab-associated hypogammaglobulinemia in autoimmune rheumatic diseases: a single-center retrospective cohort study
}

\author{
Stefanie D. Wade ${ }^{1}$ D . Vasileios C. Kyttaris ${ }^{1}$ (D)
}

Received: 15 February 2021 / Accepted: 22 March 2021 / Published online: 3 April 2021

(c) The Author(s), under exclusive licence to Springer-Verlag GmbH Germany, part of Springer Nature 2021

\begin{abstract}
B-cell targeted therapies, such as rituximab (RTX), are used widely in autoimmune rheumatic diseases (AIRD). RTX can cause hypogammaglobulinemia and predispose patients to infections. Herein, we asked whether the underlying diagnosis influences the risk for hypogammaglobulinemia in patients treated with RTX. All patients who received RTX infusions and carried a diagnosis of rheumatoid arthritis (RA), ANCA-associated vasculitis (AAV), or connective tissue disease (CTD) were included in this single-center retrospective cohort study. We used STATA® for analysis: Chi-square test was used for comparing categorical variables. Based on distribution, continuous variables were compared using the $t$ test/ANOVA or the Wilcoxon/Kruskal-Wallis tests. Of the 163 patients who received RTX for an AIRD, 60 with pre- and post- RTX immunoglobulins were analyzed. A higher incidence of post-treatment hypogammaglobulinemia was seen in AAV (45\%) compared to RA (22\%) and CTD (9.1\%) groups $(p=0.03)$. Glucocorticoid exposure of $10 \mathrm{mg}$ or more was identified as a significant risk factor for hypogammaglobulinemia. Finally, we observed a higher number of clinically significant infections per person in the AAV group than in the RA and CTD groups. We observed an increased incidence of hypogammaglobulinemia in the RTX-treated AAV group, with almost half of patients developing post-RTX hypogammaglobulinemia. The rate of infections per person was highest in the AAV group. Screening immunoglobulins were not consistently measured pre- and post-RTX. Results highlight a need for increased awareness of the role of immunoglobulin measurement before maintenance doses of RTX, especially in patients with AAV and steroid exposure.
\end{abstract}

Keywords Autoimmune disease $\cdot$ Rituximab $\cdot$ Hypogammaglobulinemia $\cdot$ Infection $\cdot$ Vaccination

\section{Introduction}

Rituximab (RTX) is a chimeric monoclonal antibody that binds the CD20 molecule on the surface of B cells and leads to $\mathrm{B}$ cell depletion. After the initial depletion, B cell reconstitution typically occurs 6-9 months post-RTX infusion,

Presented as a podium presentation at ACR Annual Meeting in November 2019. Wade S, Kyttaris V. Comparison of RituximabAssociated Hypogammaglobulinemia Rates in Patients with Systemic Rheumatologic Conditions [abstract]. Arthritis Rheumatol. 2019; 71 (suppl 10). https://acrabstracts.org/abstr act/comparison-of-rituximab-associated-hypogammaglobul inemia-rates-in-patients-with-systemic-rheumatologic-conditions/. Accessed April 30, 2020.

Vasileios C. Kyttaris

vkyttari@bidmc.harvard.edu

1 Division of Rheumatology and Clinical Immunology, Harvard Medical School, Beth Israel Deaconess Medical Center, Boston, MA, USA with their population being skewed toward naïve B cells [1]. This B-cell targeted therapy (BCTT) is FDA approved and widely used in autoimmune rheumatic diseases (AIRD) such anti-neutrophil cytoplasmic antibody (ANCA)-associated vasculitis (AAV) and rheumatoid arthritis (RA) [2-5]. RTX is also recommended by the European League Against Rheumatism as third-line treatment in systemic lupus erythematosus (SLE) and it is used off-label in patients with other forms of connective tissue disease (CTD) [6-9].

The CD20 surface molecule is lost prior to B-cell differentiation into plasma cells, the major source of circulating immunoglobulin $\mathrm{G}(\mathrm{IgG})$, and thus, effects on gamma globulin production after short courses of RTX should be a rare event. In fact, hypogammaglobulinemia, defined as reductions in circulating immunoglobulins (Ig), was not a major concern in AAV clinical trials or in early reports from RA clinical trials $[2,10,11]$. There is a paucity of data on the incidence of hypogammaglobulinemia with RTX use in CTD and a phase III trial (in SLE) assessing combination 
BCTT, with RTX and belimumab, excluded patients with low IgG levels [12-14].

Real-world experience has shown that hypogammaglobulinemia is a concern with RTX and has been associated with reduced response to vaccines, increased susceptibility to bacterial infections, and reactivation of viral infections [15]. Similar concerns are anticipated with novel BCTT that are currently under study for AIRD. Some experts recommend measuring serum immunoglobulin levels prior to initiating RTX and before repeat cycles of RTX; however, there are no consensus guidelines regarding screening for hypogammaglobulinemia in AIRD [16, 17]. Functional assessment, through measuring antibody titers, is also an important consideration in patients receiving treatment with RTX.

Risk factors for the development of hypogammaglobulinemia appear to reflect differences in dose and schedule, concomitant therapies, and underlying disease state [15, 18]. The aim of the study presented herein was to address the differences in the rate of RTX-associated hypogammaglobulinemia among RA, AAV, and CTD patients in clinical practice, and to report the incidence of infections in these patients.

\section{Materials and methods}

\section{Study design}

We performed a medical records review study in patients who received RTX for AIRD at an academic tertiary care hospital. Data was collected by cross-referencing pharmacy infusion records with billing codes for AIRD. Charts were reviewed in full and data were subsequently de-identified for analysis. Ethics committee approval was obtained with waiver of informed consent (institutional review board protocol \#: 2018P000703). This study followed the Strengthening the Reporting of Observational Studies in Epidemiology (STROBE) guideline.

\section{Inclusion and exclusion criteria}

Adult patients who received RTX infusions for RA, AAV, or CTD at BIDMC between January 1, 2002 and October 1, 2019 were included. The CTD group was a heterogeneous group composed of SLE, primary Sjögren's syndrome, overlap syndromes, anti-synthetase syndrome, mixed CTD (MCTD), and undifferentiated CTD (UCTD). Patients were excluded if they did not receive follow-up care at our institution post-RTX administration and if they received RTX for non-rheumatologic indications (such as peri-transplant or for treatment of a hematologic malignancy). Patients were also excluded if they received concomitant immunoglobulin replacement therapy (IGRT) for the treatment of their underlying AIRD; those who went on to receive IGRT for the treatment of recurrent infections were not excluded.

\section{Clinical and laboratory data}

Baseline data at initiation of RTX, including demographics, information about autoimmune diagnosis (RA, CTD, or AAV), co-morbidities (coronary artery disease or equivalent, hypertension, diabetes mellitus, chronic kidney disease, chronic liver disease, history of cancer, hypogammaglobulinemia, or chronic infection), glucocorticoid exposure, and other immunosuppressive drugs were recorded. Glucocorticoid exposure was defined as a daily steroid dose of moderate-to-high as a dose equivalent to $10 \mathrm{mg}$ or prednisone per day or more versus low, being less than $10 \mathrm{mg}$ per day at the initiation of RTX treatment. Prior and concomitant immunosuppressive drugs or DMARDS (disease-modifying anti-rheumatic drugs) including methotrexate, leflunomide, cyclophosphamide (CYC), mycophenolate, azathioprine, and tacrolimus were recorded. Quantitative $\mathrm{IgG}$, measured via enzyme-linked immune-sorbent assay (ELISA), and/or semiquantitative immunoglobulin levels, measured via serum protein electrophoresis (SPEP), were used for the determination of hypogammaglobulinemia. Hypogammaglobulinemia by ELISA was defined as serum $\operatorname{IgG}$ level below $600 \mathrm{mg} / \mathrm{dL}$ (normal range 700 to $1600 \mathrm{mg} / \mathrm{dL}$ ). Immunoglobulins were measured according to local practice. When IgG levels were measured more than once within a short period of time, the first measurement was used.

Safety data regarding clinically significant infections were abstracted from clinician notes, microbiology results, and antibiotic prescriptions. Serious infection events (SIE), defined as infections warranting hospital admission, the use of intravenous antibiotics, or death, were recorded. We also recorded clinically significant infections which besides SIE, included: episodes of pneumonia confirmed radiographically and/or via bronchoscopy; complicated urinary tract infections (UTI); infections requiring hospitalization that were treated with oral antibiotics; severe systemic viral infections including disseminated varicella zoster virus (VZV) and disseminated herpes simplex virus (HSV). Infections within the first year of RTX use were recorded for all patients with pre- and post-RTX IgG measurements. Infections from the full cohort, regardless of immunoglobulin measurements, were recorded and described separately. 


\section{Primary outcome}

The incidence of new-onset hypogammaglobulinemia in RA, $\mathrm{AAV}$, and CTD patients.

\section{Statistical analysis}

Analysis was performed using STATA®. We used the Shapiro-Wilk test for normal distribution of continuous variables. Normally distributed continuous variables were compared using t test (dichotomous predictor) or ANOVA (categorical predictor). Non-normally distributed continuous variables were compared using the Wilcoxon (dichotomous predictor) or the Kruskal-Wallis (categorical predictor) test. We used the Chi-square test to compare dichotomous variables.

\section{Results}

\section{Patient characteristics}

We screened 302 patients with AIRD who were treated with RTX; 163 of these patients received RTX for RA, AAV, or a CTD (Fig. 1). A total of 114 of the 163 patients (69.9\%) had baseline screening immunoglobulin measurements. A total of 80 out of 163 patients (49.1\%) had immunoglobulin assessments after initiation of treatment with RTX. Sixty out of the 163 patients had IgG levels pre- and post-RTX (Fig. 1). Baseline patient characteristics are listed in Table 1 and were typical of what would be expected in a cohort of patients with autoimmune diseases. There were three

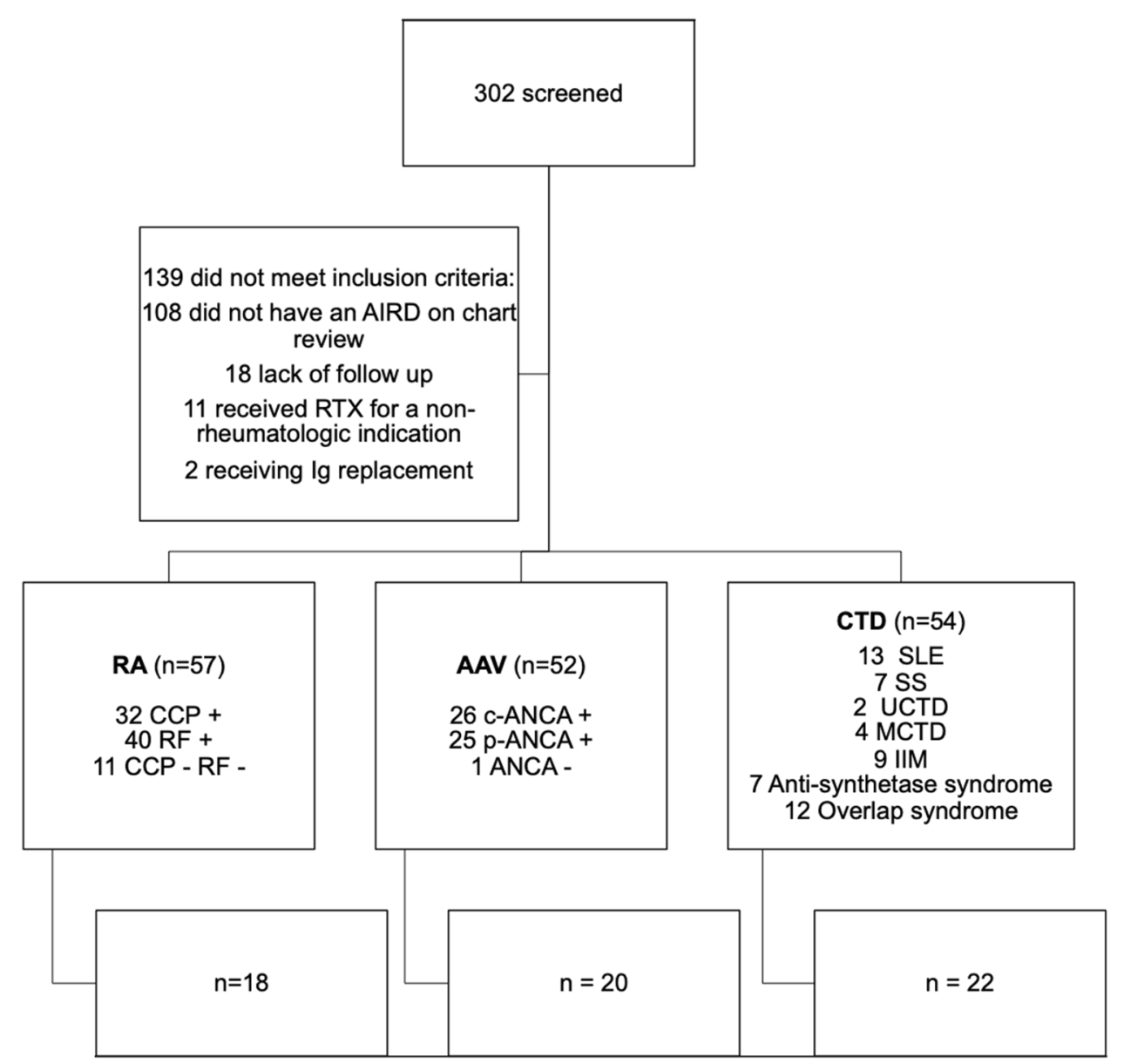

Fig. 1 Patients with AIRD treated with rituximab were included in the study. We excluded patients without a well-established diagnosis of AAV, RA, or CTD, after comprehensive chart review, those without follow-up at our center, those who received RTX for a non-rheumatologic indication, and those who received concomitant Ig replacement for the treatment of their underlying disease (second row). Of the remaining patients, we analyzed those who had IgG measurements before and after rituximab treatment (fourth row); however, we reported on infections for the whole cohort (third row). AIRD auto- immune rheumatic disease, $A A V$ anti-neutrophil cytoplasmic antibody (ANCA)-associated vasculitis, $C$-ANCA cytoplasmic-ANCA, $C C P$ cyclic citrullinated peptide, $C T D$ connective tissue disease, IIM idiopathic inflammatory myopathy, $I g$ immunoglobulin, $M C T D$ mixed connective tissue disease, $P$-ANCA perinuclear-ANCA, $R A$ rheumatoid arthritis, $R F$ rheumatoid factor, $S L E$ systemic lupus erythematosus, SS Sjogren's syndrome, UCTD undifferentiated connective tissue disease 
Table 1 Characteristics of patients treated with rituximab

\begin{tabular}{|c|c|c|c|c|}
\hline & All patients $(n=163)$ & RA $(n=57)$ & $\operatorname{AAV}(n=52)$ & $\mathrm{CTD}(n=54)$ \\
\hline Gender $n(\%$ female $)$ & $122(74.8)$ & $44(77.2)$ & $34(65.4)$ & $44(81.5)$ \\
\hline Race (\% Caucasian) & 161(71.4) & $44(77.2)$ & $42(82.4)$ & $29(53.7)$ \\
\hline Age at first RTX, mean (SD) & $55.9(15.5)$ & $56.4(13.6)$ & $61.6(15.5)$ & $49.9(15.3)$ \\
\hline Disease duration (years) at first RTX, median (IQR) & $3(0,10)$ & $10(4,18)$ & $0(0,1)$ & $2(1,6)$ \\
\hline \# Co-morbidities, median (IQR) & $2(1,3)$ & $2(1,3)$ & $3(2,4)$ & $2(1,3)$ \\
\hline \# Prior conventional DMARD, median (IQR) & $2(1,2)$ & $2(1,2)$ & $0(0,1)$ & $2(1,3)$ \\
\hline \# Prior biologic DMARD, median (IQR) & $0(0,2)$ & $2(1,3)$ & $0(0,0)$ & $0(0,0)$ \\
\hline PJP prophylaxis, $n(\%)$ & $73(45.6)$ & $2(3.5)$ & $47(94.0)$ & $23(42.6)$ \\
\hline Influenza vaccine, $n(\%)$ & $102(81.6)$ & $33(75.0)$ & $40(85.1)$ & $29(85.3)$ \\
\hline PPSV23, $n(\%)$ & $84(84.8)$ & $31(86.1)$ & $30(81.1)$ & $21(80.8)$ \\
\hline PCV13, $n(\%)$ & $49(50.6)$ & $18(50.0)$ & $12(38.7)$ & $15(68.2)$ \\
\hline Past cyclophosphamide, $n(\%)$ & $26(16.0)$ & $1(1.8)$ & $20(38.5)$ & $5(9.3)$ \\
\hline Concomitant DMARD (yes/no), $n(\%)$ & $61(37.4)$ & $24(42.1)$ & $7(13.5)$ & $32(59.3)$ \\
\hline Steroids $\geq 10 \mathrm{mg} /$ day, $n(\%)$ & $101(62.0)$ & $16(28.1)$ & $51(98.1)$ & $35(64.8)$ \\
\hline Duration of RTX (m), median (IQR) & $11(1,30)$ & $19(6,56)$ & $7(1,24.5)$ & $1(1,22)$ \\
\hline Pre-RTX Ig Assessment, $n(\%)$ & $114(69.9)$ & $38(66.7)$ & $32(61.5)$ & $44(81.5)$ \\
\hline Pre-RTX hypogammaglobulinemia n, (\%) & $3(2.6)$ & $1(2.6)$ & $1(1.9)$ & $1(1.9)$ \\
\hline Post-RTX Ig Assessment, $n(\%)$ & $80(49.1)$ & $23(40.4)$ & $29(55.8)$ & $28(51.9)$ \\
\hline Post-RTX hypogammaglobulinemia, $n(\%)$ & $17(21.2)$ & $5(21.7)$ & $11(37.9)$ & $3(10.7)$ \\
\hline Cumulative RTX at post RTX Ig Assessment (g), median (IQR) & $3(2,5)$ & $3(2,6.8)$ & $3(2.4,4.2)$ & $2.95(2,5.5)$ \\
\hline Pre $\&$ post-RTX Ig Assessment, $n(\%)$ & $60(36.8)$ & $18(31.6)$ & $20(38.5)$ & $22(40.7)$ \\
\hline
\end{tabular}

Timing of vaccines was variable in relation to first RTX dose. Co-morbidities included coronary artery disease or equivalent, hypertension, diabetes mellitus, chronic kidney disease, chronic liver disease, or malignancy; cumulative RTX dose was calculated from the time of the first RTX infusion until the time of the post-RTX IgG measurement

Ig Immunoglobulin, PCV13 pneumococcal conjugate vaccine, PJP pneumocystis jirovecii pneumonia, PPSV23: pneumococcal polysaccharide vaccine. Normally distributed continuous variables are presented as mean (standard deviation-SD). Non-normally distributed continuous variables are presented as median (inter quartile range-IQR)

patients with hypogammaglobulinemia prior to RTX infusion who were not included in the analysis.

\section{Primary outcome: hypogammaglobulinemia incidence by disease}

We analyzed the 60 patients who had immunoglobulins measured both prior to initiation of RTX and post-treatment with RTX (Table 2). Median time frame (IQR) of immunoglobulin measurement was 4 months (IQR:1, 12 months) pre- and 8.5 months (IQR: 3, 18 months) post-RTX administration. The total duration of follow-up post last immunoglobulin measurement was 13 months (IQR: 3, 26.5 months). A total of 15 out of $60(25.0 \%)$ patients developed newonset hypogammaglobulinemia post-treatment with RTX. The incidence of hypogammaglobulinemia was significantly higher in the AAV patients (45.0\%) compared to RA (22.2\%) and CTD (9.1\%) patients (Chi-square test $p=0.03$ ) (Fig. 2). No patients had IgG levels less than $100 \mathrm{mg} / \mathrm{dL}$.

\section{Characteristics of hypogammaglobulinemic patients}

We further analyzed patients according to whether or not they developed hypogammaglobulinemia (Table 3). Exposure to moderate-to-high doses of steroids (defined as prednisone daily equivalent dose $\geq 10 \mathrm{mg}$ per day) at RTX onset was associated with incident hypogammaglobulinemia (Chi-square test $p=0.045$ ). The association between previous treatment with CYC and hypogammaglobulinemia was not statistically significant. When analyzed by underlying disease, almost all hypogammaglobulinemic patients were on high doses of steroids: 9/9 AAV, 2/2 CTD, and 2/4 RA patients.

\section{Infections}

A total of 12 out of $60(20.0 \%)$ patients had 13 clinically significant infections within the first year of RTX (21.7/100 
Table 2 Characteristics of patients with pre- and post-RTX immunoglobulin measurements
Fig. 2 Incidence of rituximabassociated hypogammaglobulinemia across RA, AAV, and CTD patients. $A A V$ ANCAassociated vasculitis, $C T D$ connective tissue disease, $R A$ rheumatoid arthritis. The Chisquare test was used for among groups' comparisons

\begin{tabular}{llllr}
\hline Patients $(n=60)$ & RA $(n=18)$ & AAV $(n=20)$ & CTD $(n=22)$ & $p$ value \\
\hline Gender (\% female) & $12(66.7)$ & $14(70.0)$ & $19(86.4)$ & 0.29 \\
Race (\% Caucasian) & $14(77.8)$ & $12(63.0)$ & $15(68.1)$ & 0.62 \\
$\begin{array}{l}\text { Disease duration (years) at first RTX, } \\
\quad \text { median (IQR) }\end{array}$ & $6(3,22)$ & $1(0,2.5)$ & $2(1,6)$ & $<0.01$ \\
Age at first RTX, mean (SD) & $62.2(13.1)$ & $58.0(18.3)$ & $53(11.5))$ & 0.14 \\
Cumulative RTX (g), median (IQR) & $2.5(2,6.8)$ & $2.95(2.4,4.1)$ & $2.85(2,4)$ & 0.71 \\
\# Co-morbidities, median (IQR) & $2(1,3)$ & $2.5(1,4)$ & $2(1,3)$ & 0.64 \\
Past cyclophosphamide, $n(\%)$ & $0(0)$ & $7(35.0)$ & $1(4.5)$ & $<0.01$ \\
Concomitant DMARD (yes/no) $n(\%)$ & $7(38.9)$ & $3(15.0)$ & $13(59.0)$ & 0.01 \\
Steroids $\geq 10$ mg/day, $n(\%)$ & $7(38.9)$ & $20(100.0)$ & $13(59.1)$ & $<0.01$ \\
\hline
\end{tabular}

Cumulative RTX dose was calculated from the time of the first RTX infusion until the time of the postRTX IgG measurement. Co-morbidities included coronary artery disease or equivalent, hypertension, diabetes mellitus, chronic kidney disease, chronic liver disease, or malignancy. Normally distributed continuous variables are presented as mean (Standard Deviation-SD). Non-normally distributed continuous variables are presented as median (Inter Quartile Range-IQR). Normally distributed variables were compared using ANOVA. Non-normally distributed variables were compared using the Kruskal-Wallis test

The Chi-square was used to compare dichotomous variables

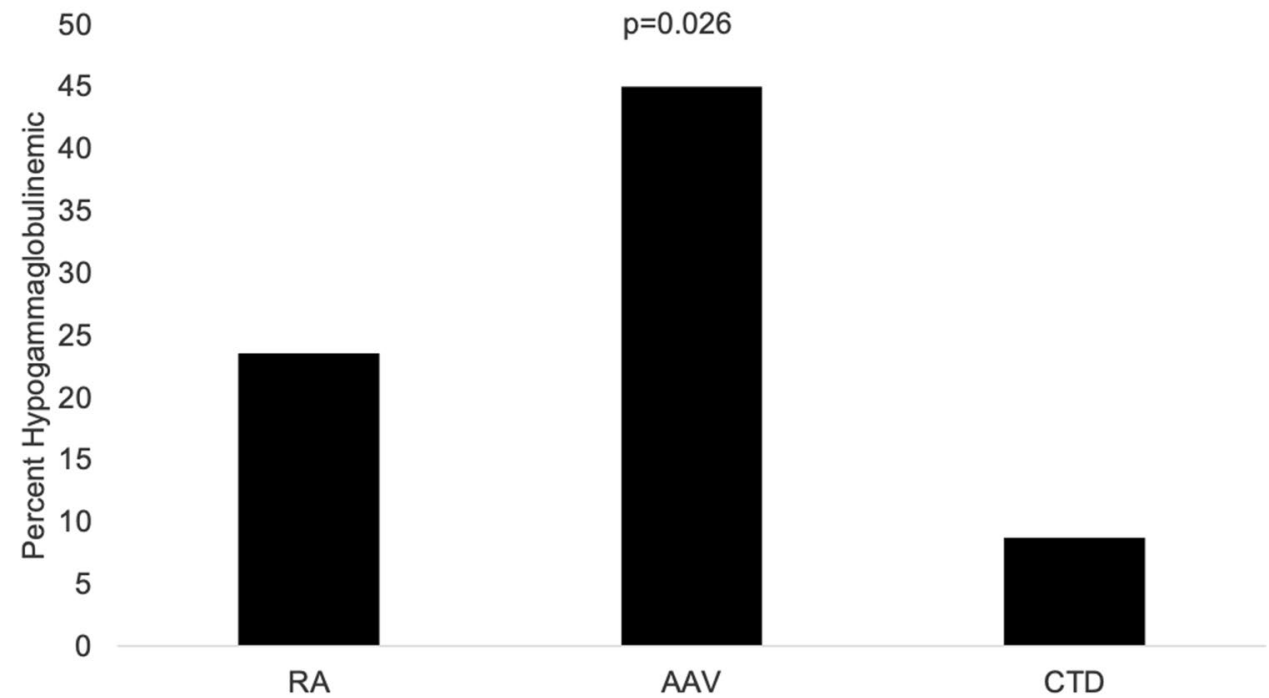

PY). There were 0.35 infections per person in the AAV group versus 0.17 and 0.14 infections per person in the RA and CTD groups. IgG levels were not routinely evaluated during infectious episodes and only 8 patients had immunoglobulin measurements within 6 months of their documented infection. Infections were not significantly associated with new hypogammaglobulinemia (Chi-square test $p=0.18$ ), although $42 \%$ (5 out of 12) patients with infections had hypogammaglobulinemia at some point over the course of their treatment with RTX. Lymphopenia was seen in $42 \%$ of the patients at the time of infection $(n=5)$, but there was no associated neutropenia. Two out of the 12 patients with low IgG were ultimately treated with IGRT due to recurrent infections.

Additional two patients received IGRT from the larger cohort; however, they were not included in the above analysis as they did not have immunoglobulin measurements prior to RTX initiation, so we could not establish whether this was new onset hypogammaglobulinemia post-RTX. Of the four patients who received IGRT in the setting of hypogammaglobulinemia, indications included recurrent infections (three patients) and severe persistent hypogammaglobulinemia with a further need for RTX (one patient). 
Table 3 Features of patients who developed rituximab-associated hypogammaglobulinemia compared to patients without hypogammaglobulinemia

\begin{tabular}{lllr}
\hline & $\begin{array}{l}\text { Non-hypogammaglobu- } \\
\text { linemic }\end{array}$ & $\begin{array}{l}\text { Hypogammaglobu- } \\
\text { linemic }\end{array}$ & $p$ value \\
\hline Patients, $n$ & 45 & 15 & \\
Female gender, $n(\%)$ & $34(75.6)$ & $11(73.3)$ & 0.86 \\
Caucasian Race, $n(\%)$ & $29(64 \%)$ & $12(86 \%)$ & 0.13 \\
Age at first RTX, mean (SD) & $56.2(15.4)$ & $61.1(12.5)$ & 0.27 \\
\# Co-morbidities, median (IQR) & $2(1,3)$ & $3(1,4)$ & 0.34 \\
\# Prior DMARDS median (IQR) & $2(1,3)$ & $1(0,4)$ & 0.81 \\
Past cyclophosphamide, $n(\%)$ & $5(11.1)$ & $3(20.0)$ & 0.38 \\
Concomitant DMARD, $n(\%)$ & $19(42.2)$ & $4(26.7)$ & 0.28 \\
Cumulative RTX (g), median (IQR) & $2.8(2,4)$ & $4(2.4,6)$ & 0.18 \\
Prednisone $\geq 10$ mg/day, $n(\%)$ & $27(60.0)$ & $13(86.7)$ & $<0.05$ \\
\hline
\end{tabular}

Concomitant DMARD's included simultaneous treatment with methotrexate, leflunomide, mycophenolate mofetil, or azathioprine. Cumulative RTX dosing was calculated from the time of the first RTX infusion until the time of the post-RTX IgG measurement. Normally distributed continuous variables are presented as mean (Standard Deviation-SD). Non-normally distributed continuous variables are presented as median (Inter Quartile Range-IQR). Normally distributed variables were compared using $t$ test. Nonnormally distributed variables were compared using the Wilcoxon test

The Chi-square was used to compare dichotomous variables
We separately evaluated the entire cohort of 163 RTXtreated patients, including patients without immunoglobulin measurements, totaling 385 PY. Infection events were recorded from RTX initiation until 6 months following their last recorded RTX dose. A total of 29 (17.8\%) of the patients had 38 clinically significant infectious episodes (9.9/100 PY). A total of 19 out of the 38 clinically significant infections were categorized as SIE resulting in an SIE rate of 4.9/100 PY. The most common clinically significant infections included pneumonia ( $n=14 ; 11$ bacterial, 3 viral), followed by sepsis from various sources $(n=8)$ and soft-tissue infections $(n=6)$. Viral infections with HSV, VZV, and CMV were common $(n=6)$ with CMV contributing to 2 of the episodes of pneumonia and $1 \mathrm{HSV}$ case leading to severe keratitis. There were 3 cases of nontuberculous mycobacterial (NTM) infections: 1 case of recurrent MAI infection in a patient with prior CYC exposure and 2 patients with new NTM infections-all three patients were receiving concomitant high-dose glucocorticoids for the treatment of AAV. There were seven infection-related deaths. Two patients died from pneumonia: one bacterial pneumonia and one with CMV pneumonia. Four patients died from sepsis from different sources. There did not appear to be any significant differences between the baseline characteristics of the patients who had immunoglobulin measurements prior to and after treatment with RTX from the remainder of the cohort.

\section{Discussion}

Almost half $(45 \%)$ of patients with AAV in our cohort developed new hypogammaglobulinemia after treatment with RTX. The incidence of hypogammaglobulinemia was significantly lower in RA and CTD patients. These findings suggest that AAV confers greater risk for hypogammaglobulinemia than RA and CTD patients receiving RTX. Our findings are similar to a study that identified a higher incidence of hypogammaglobulinemia in AAV versus RA and CTD groups [19]. It has been proposed that prolonged median time of B-cell depletion in AAV patients compared to RA and CTD groups may lead to increased hypogammaglobulinemia incidence; however, the mechanism behind this difference has not been established [20]. Results from yet another study looking at patients from the United Kingdom with AIRD receiving RTX found both glucocorticoid exposure and CYC exposure to be associated with increased incidence of hypogammaglobulinemia [20]. We identified high glucocorticoid exposure as a risk factor for hypogammaglobulinemia in our cohort; however, the majority of patients who developed hypogammaglobulinemia in our cohort did not have prior CYC exposure. Trends toward increased use of RTX as a first-line agent in AAV will likely make this less of an issue in the future. A retrospective cohort study from the United States identified baseline IgG level prior to maintenance RTX as the sole predictor of developing significant hypogammaglobulinemia during maintenance for AAV [21]. One-fifth of RA patients in our cohort developed hypogammaglobulinemia. We did not see an increased risk of hypogammaglobulinemia in patients receiving concomitant conventional DMARDs, which is in keeping with RA literature. Observational data show that low baseline serum IgG levels in RA patients are an important risk factor for developing persistent hypogammaglobulinemia [22]. Very few CTD patients developed IgG hypogammaglobulinemia in our cohort. This is in keeping with a retrospective study 
in SLE patients who were followed longitudinally during RTX therapy [23].

We observed a numerically higher rate of infections per person during the first year of RTX administration in AAV patients compared to RA and CTD patients. Those with hypogammaglobulinemia developed both bacterial and viral infections, and, in AAV patients on moderate-to-high doses of steroids, NTM infections. In our cohort, the majority of severe infections were bacterial or viral pneumonias that may be preventable with appropriate vaccination. While gastrointestinal infections were rare, there was one case of sepsis secondary to severe colitis. We did not find any cases of severe influenza infection in our cohort, which may reflect high rates of influenza vaccination. Rates of pneumonia vaccination were variable in our cohort and we did not record vaccination rates for the VZV. Subsequent to our analysis, preliminary research into AIRD patients on RTX infected with the novel SARS-CoV2 virus may have higher rates of hospitalization and death [24].

A recent longitudinal study of 700 patients with rheumatic diseases identified higher corticosteroid doses and low IgG levels as predictors of SIE in patients receiving RTX, with an SIE of 21.3/100 PY in those with persistent IgG hypogammaglobulinemia versus $9.7 / 100 \mathrm{PY}$ in those without IgG hypogammaglobulinemia [25]. Increasing availability of potent oral antibiotics and non-inferiority of oral antibiotics in certain infections may herald a trend toward decreased use of intravenous antibiotics [26, 27]. As a result of these trends, the SIE rate potentially underestimates the risk of serious infections in rheumatologic patients receiving RTX. There have not been any randomized trials looking at RTX in CTD-associated ILD; however, observational data show that the most frequent reason for RTX discontinuation is infection [28].

A strength of our study was that charts were reviewed in full which allowed for granular assessment of the types of infections seen in patients with AIRD during treatment with RTX. Limitations include the retrospective nature of the study, as we were unable to control the timing of IgG levels or consistently describe whether hypogammaglobulinemia was transient versus persistent. Due to sample size limitations, we were unable to identify predictors of hypogammaglobulinemia by individual disease state. Analysis of infections post-RTX was similarly limited by the sample size and the fact that immunoglobulins were not consistently measured at the time of infection. Relevant infections were captured for patients who tended to be admitted within our institution; however, there may be missing events for patients receiving primary care outside of our center.

\section{Considerations regarding immunoglobulin measurement}

We limited our primary analysis to patients with new hypogammaglobulinemia after RTX use; however, observational studies support a role for screening with quantitative immunoglobulins prior to beginning BCTT to identify patients at risk for persistent hypogammaglobulinemia and infections [23, 29, 30]. Our results support the role of routine immunoglobulin monitoring for high-risk patients, such as patients with $\mathrm{AAV}$ and those requiring high doses of steroids. An expert panel from the UK recommends screening with immunoglobulins every 6-12 months for the duration of BCTT; however, this has not been universally adopted in clinical practice [18]. Immunoglobulins were measured pre- and post-RTX in less than half of our cohort, which is comparable to other North American cohorts [31]. Rheumatologists would likely benefit from education regarding the rationale for screening immunoglobulins in AIRD patients receiving RTX.

\section{Considerations for BCTT in patients with hypogammaglobulinemia}

The detection of hypogammaglobulinemia at pre-RTX screening should not necessarily delay treatment, particularly in patients with organ or life-threatening disease. This is in line with a panel of experts from the United Kingdom, who recommend making RTX treatment decisions based on an individual risk-benefit analysis [18]. Decisions regarding RTX use should be based on disease severity, infection history, current infectious risk factors, degree of hypogammaglobulinemia, the availability of IGRT, and the presence of alternative treatment options. For maintenance in AAV, using a $500 \mathrm{mg}$ over a $1000 \mathrm{mg}$ maintenance dose appears to confer less risk for hypogammaglobulinemia [32]. Additionally, long-term follow-up from the Autoimmunity and Rituximab Registry in RA reported a higher rate of serious infections in patients receiving higher maintenance doses (i.e., $1000 \mathrm{mg} \times 2$ doses) compared to those receiving lower doses [33]. In severe disease, RTX dose and schedule could potentially continue unchanged with close monitoring and consideration of IGRT if there are clinically significant or severe infections. Providers should educate patients on the risk of persistent hypogammaglobulinemia and serious infections with BCTT to facilitate shared decision-making. RTX-related hypogammaglobulinemia in AIRD was transient in half of cases in one study, highlighting a role for rechecking serum IgG prior to subsequent cycles of RTX [34]. 


\section{Considerations for vaccination during BCTT}

Lack of a physician's recommendation has been identified as an important predictor of failure to receive vaccination [35]. Since patients receiving BCTT have a high risk of developing vaccine-preventable diseases, rheumatologists should be vigilant about assessing vaccination status annually in these patients [36]. BCTT can be associated with impaired vaccine responses [37], and thus, pneumonia and influenza vaccines should be administered prior to initiating RTX when possible [36]. EULAR guidelines for non-immunized patients on BCTT recommend administering vaccines at least 6 months after receiving their last RTX infusion, and 4 weeks prior to their subsequent course, as this is the time period during which peak vaccine response rates are presumed to occur $[36,38]$. Vaccination outside of this time window may be associated with suboptimal vaccination response and functional assessment of vaccine response may be reasonable. It is anticipated that these recommendations will hold true for vaccination against the novel SARS-CoV2 infection; however, further research is needed. Herpes zoster and toxoid tetanus vaccination should be considered according to guideline recommendations and, in case of wounds at risk of tetanus, passive immunization with anti-tetanus-Ig should be considered [36].

\section{Role for prophylactic antibiotics}

Data suggest that effector B cells can influence T-cell responses to pathogens [39]. Thus, while RTX does not cause global immunosuppression, it may contribute to the multifactorial risk for opportunistic infections, such as pneumocystis jirovecii pneumonia (PJP). We did not find any cases of PJP in our cohort, which likely reflects the rarity of this complication as well as high rates of pneumocystis prophylaxis in AAV patients in our cohort. Unless contraindicated, PJP prophylaxis is recommended in all AAV patients receiving RTX and in CTD patients receiving RTX plus another immunosuppressant drug.

\section{Role for prophylactic antivirals}

Physicians are generally encouraged to minimize the use of RTX in patients with a history of viral hepatitis. In those who do require treatment with RTX, there is an established role for antiviral therapy in patients with a history of hepatitis B and hepatitis C. Clear guidelines exist for hematologic patients undergoing bone marrow transplant regarding the use of empiric prophylaxis against other common viruses, such as human herpesviruses (HHV) [40]. More research is needed to establish whether there is a role for empiric prophylaxis for AIRD patients during treatment with BCTT in those with serologic evidence of past exposure to HHV.
Role for immunoglobulin replacement therapy (IGRT)

There is no agreed-upon threshold IgG level in AIRD to warrant IGRT; however, recommendations for IGRT in BCTT-related hypogammaglobulinemia were recently published and may provide helpful guidance for the management of hypogammaglobulinemia [41]. Consideration of IGRT may be necessary for those with recurrent infections, particularly when continued RTX is important to treat the underlying AIRD [41]. If hypogammaglobulinemia is identified, then further evaluation of antibody responses to vaccines and close monitoring for infections may be helpful for identifying patients who might benefit from IGRT. A retrospective study looking at patients with multi-system AIRD treated with RTX found that $4.2 \%$ of their cohort received IGRT for recurrent infections [34]. Decreasing IgG levels increased the odds ratio of receiving IGRT for hypogammaglobulinemia. In patients with secondary immunodeficiency after RTX, there appear to be two groups of patients: one subset who may recover immunoglobulins and B cell counts versus another subset with long-lasting depletion of memory B cells and immunoglobulin levels [37]. This concept is highlighted in a report of 16 patients who received IGRT for RTX-related hypogammaglobulinemia: IGRT was successfully tapered off in two patients; whilst, the majority of patients required an extended duration of IGRT [37].

\section{Conclusions}

In our cohort, AAV patients were more likely to develop hypogammaglobulinemia and infectious complications than RA and CTD patients in the first year of RTX administration. Immunoglobulins were not consistently measured pre- and post-RTX despite increasing evidence that IgG hypogammaglobulinemia is associated with infectious complications. The majority of infections in our cohort included viral and bacterial pneumonias, highlighting the importance of annual vaccine status assessment in AIRD patients receiving BCTT. Our results suggest that in high-risk patients, such as AAV patients and those requiring high doses of steroids, there may be a role for regular monitoring of $\mathrm{IgG}$ levels in patients receiving BCTT regardless of pre-RTX IgG levels. Additional prospective studies are needed to explore the potential increase in susceptibility to hypogammaglobulinemia in AAV patients during treatment with BCTT and to determine long-term outcomes in these patients.

Author contributions All authors contributed to the study conception and design. Material preparation and data collection were performed by SDW and data analysis was performed by VCK. The first draft of 
the manuscript was written by SDW, and all authors commented on previous versions of the manuscript and read and approved the final manuscript.

Funding This study did not receive funding.

Availability of data and materials Deidentified source data can be made available upon request.

\section{Declarations}

Conflict of interest SDW: None. VCK: No direct conflicts of interest. Dr. Kyttaris has received consulting fees from GlaxoSmithKline and Exagen Diagnostics for work unrelated to this work.

Ethical standards This study was approved by the appropriate ethics committee and has therefore been performed in accordance with the ethical standards laid down in the 1964 Declaration of Helsinki and its later amendments.

Ethics approval IRB approval was obtained from Beth Israel Deaconess Medical Center.

Consent for publication The manuscript has been read and approved for publication by all named authors.

\section{References}

1. Anolik JH, Friedberg JW, Zheng B et al (2007) B cell reconstitution after rituximab treatment of lymphoma recapitulates B cell ontogeny. Clin Immunol 122(2):139-145

2. Edwards JC, Szczepanski L, Szechinski J et al (2004) Efficacy of B-cell-targeted therapy with rituximab in patients with rheumatoid arthritis. N Engl J Med 350(25):2572-2581

3. Cohen SB, Emery P, Greenwald MW et al (2006) Rituximab for rheumatoid arthritis refractory to anti-tumor necrosis factor therapy: Results of a multicenter, randomized, double-blind, placebocontrolled, phase III trial evaluating primary efficacy and safety at twenty-four weeks. Arthritis Rheum 54(9):2793-2806

4. Emery P, Fleischmann R, Filipowicz-Sosnowska A et al (2006) The efficacy and safety of rituximab in patients with active rheumatoid arthritis despite methotrexate treatment: results of a phase IIB randomized, double-blind, placebo-controlled, dose-ranging trial. Arthritis Rheum 54(5):1390-1400

5. Stone JH, Merkel PA, Spiera R et al (2010) Rituximab versus cyclophosphamide for ANCA-associated vasculitis. N Engl J Med 363(3):221-232

6. Fanouriakis A, Kostopoulou M, Alunno A et al (2019) 2019 update of the EULAR recommendations for the management of systemic lupus erythematosus. Ann Rheum Dis 78(6):736-745

7. Diaz-Lagares C, Croca S, Sangle S et al (2012) Efficacy of rituximab in 164 patients with biopsy-proven lupus nephritis: pooled data from European cohorts. Autoimmun Rev 11(5):357-364

8. Boletis JN, Marinaki S, Skalioti C, Lionaki SS, Iniotaki A, Sfikakis PP (2009) Rituximab and mycophenolate mofetil for relapsing proliferative lupus nephritis: a long-term prospective study. Nephrol Dial Transplant 24(7):2157-2160

9. Terrier B, Amoura Z, Ravaud P et al (2010) Safety and efficacy of rituximab in systemic lupus erythematosus: results from 136 patients from the French autoimmunity and rituximab registry. Arthritis Rheum 62(8):2458-2466
10. Specks U, Merkel PA, Seo P et al (2013) Efficacy of remissioninduction regimens for ANCA-associated vasculitis. N Engl J Med 369(5):417-427

11. Guillevin L, Pagnoux C, Karras A et al (2014) Rituximab versus azathioprine for maintenance in ANCA-associated vasculitis. $\mathrm{N}$ Engl J Med 371(19):1771-1780

12. Merrill JT, Neuwelt CM, Wallace DJ et al (2010) Efficacy and safety of rituximab in moderately-to-severely active systemic lupus erythematosus: the randomized, double-blind, phase II/ III systemic lupus erythematosus evaluation of rituximab trial. Arthritis Rheum 62(1):222-233

13. Rovin BH, Furie R, Latinis K et al (2012) Efficacy and safety of rituximab in patients with active proliferative lupus nephritis: the Lupus Nephritis Assessment with Rituximab study. Arthritis Rheum 64(4):1215-1226

14. Teng YKO, Bruce IN, Diamond B et al (2019) Phase III, multicentre, randomized, double-blind, placebo-controlled, 104-week study of subcutaneous belimumab administered in combination with rituximab in adults with systemic lupus erythematosus (SLE): BLISS-BELIEVE study protocol. BMJ Open 9(3):e025687

15. Christou EAA, Giardino G, Worth A, Ladomenou F (2017) Risk factors predisposing to the development of hypogammaglobulinemia and infections post-Rituximab. Int Rev Immunol 36(6):352-359

16. Buch MH, Smolen JS, Betteridge N et al (2011) Updated consensus statement on the use of rituximab in patients with rheumatoid arthritis. Ann Rheum Dis 70(6):909-920

17. Wijetilleka S, Jayne DR, Mukhtyar C et al (2019) Recommendations for the management of secondary hypogammaglobulinaemia due to B cell targeted therapies in autoimmune rheumatic diseases. Rheumatology 58(5):889-896

18. Fernandez-Castro M, Mellor-Pita S, Citores MJ et al (2007) Common variable immunodeficiency in systemic lupus erythematosus. Semin Arthritis Rheum 36(4):238-245

19. Thiel J, Rizzi M, Engesser M et al (2017) B cell repopulation kinetics after rituximab treatment in ANCA-associated vasculitides compared to rheumatoid arthritis, and connective tissue diseases: a longitudinal observational study on 120 patients. Arthritis Res Ther 19(1):101

20. Marco H, Smith RM, Jones RB et al (2014) The effect of rituximab therapy on immunoglobulin levels in patients with multisystem autoimmune disease. BMC Musculoskelet Disord 15:178

21. Cortazar FB, Pendergraft WF 3rd, Wenger J, Owens CT, Laliberte K, Niles JL (2017) Effect of continuous B cell depletion with rituximab on pathogenic autoantibodies and total IgG levels in antineutrophil cytoplasmic antibody-associated vasculitis. Arthritis Rheumatol 69(5):1045-1053

22. De La Torre I, Leandro MJ, Valor L, Becerra E, Edwards JC, Cambridge G (2012) Total serum immunoglobulin levels in patients with RA after multiple B-cell depletion cycles based on rituximab: relationship with B-cell kinetics. Rheumatology 51(5):833-840

23. Reddy V, Martinez L, Isenberg DA, Leandro MJ, Cambridge G (2017) Pragmatic treatment of patients with systemic lupus erythematosus with rituximab: long-term effects on serum immunoglobulins. Arthritis Care Res (Hoboken) 69(6):857-866

24. García-Fernández A, López-Gutiérrez F, Loarce-Martos J et al (2020) Cohort of rheumatic patients treated with rituximab and COVID-19: Does rituximab treatment increases the severity of SARS-COV2 Infection? [abstract]. Arthritis Rheumatol 72(suppl): 10

25. Md Yusof MY, Vital EM, McElvenny DM et al (2019) Predicting Severe infection and effects of hypogammaglobulinemia during therapy with rituximab in rheumatic and musculoskeletal diseases. Arthritis Rheumatol 71(11):1812-1823 
26. Iversen K, Ihlemann N, Gill SU et al (2019) Partial oral versus intravenous antibiotic treatment of endocarditis. N Engl J Med 380(5):415-424

27. Li HK, Rombach I, Zambellas R et al (2019) Oral versus intravenous antibiotics for bone and joint infection. N Engl J Med 380(5):425-436

28. Duarte AC, Cordeiro A, Fernandes BM et al (2019) Rituximab in connective tissue disease-associated interstitial lung disease. Clin Rheumatol 38(7):2001-2009

29. Boleto G, Avouac J, Wipff J et al (2018) Predictors of hypogammaglobulinemia during rituximab maintenance therapy in rheumatoid arthritis: a 12-year longitudinal multi-center study. Semin Arthritis Rheum 48(2):149-154

30. Gottenberg JE, Ravaud P, Bardin T et al (2010) Risk factors for severe infections in patients with rheumatoid arthritis treated with rituximab in the autoimmunity and rituximab registry. Arthritis Rheum 62(9):2625-2632

31. Barmettler S, Ong MS, Farmer JR, Choi H, Walter J (2018) Association of immunoglobulin levels, infectious risk, and mortality with rituximab and hypogammaglobulinemia. JAMA Netw Open 1(7):e184169

32. Besada E, Koldingsnes W, Nossent JC (2014) Serum immunoglobulin levels and risk factors for hypogammaglobulinaemia during long-term maintenance therapy with rituximab in patients with granulomatosis with polyangiitis. Rheumatology 53(10):1818-1824

33. Henry J, Gottenberg JE, Rouanet $S$ et al (2018) Doses of rituximab for retreatment in rheumatoid arthritis: influence on maintenance and risk of serious infection. Rheumatology 57(3):538-547

34. Roberts DM, Jones RB, Smith RM et al (2015) Rituximabassociated hypogammaglobulinemia: incidence, predictors and outcomes in patients with multi-system autoimmune disease. J Autoimmun 57:60-65

35. Assala M, Groh M, Blanche P et al (2017) Pneumococcal and influenza vaccination rates in patients treated with corticosteroids and/or immunosuppressive therapies for systemic autoimmune diseases: a cross-sectional study. Jt Bone Spine 84(3):365-366

36. Furer V, Rondaan C, Heijstek MW et al (2020) 2019 update of EULAR recommendations for vaccination in adult patients with autoimmune inflammatory rheumatic diseases. Ann Rheum Dis 79(1):39-52

37. Barmettler S, Price C (2015) Continuing IgG replacement therapy for hypogammaglobulinemia after rituximab-for how long? J Allergy Clin Immunol 136(5):1407-1409

38. Papp KA, Haraoui B, Kumar D et al (2019) Vaccination guidelines for patients with immune-mediated disorders taking immunosuppressive therapies: executive summary. J Rheumatol 46(7):751-754

39. Lund FE, Randall TD (2010) Effector and regulatory B cells: modulators of CD4+ T cell immunity. Nat Rev Immunol 10(4):236-247

40. Tomblyn M, Chiller T, Einsele H et al (2009) Guidelines for preventing infectious complications among hematopoietic cell transplantation recipients: a global perspective. Biol Blood Marrow Transplant 15(10):1143-1238

41. Wijetilleka S, Mukhtyar C, Jayne D et al (2019) Immunoglobulin replacement for secondary immunodeficiency after B-cell targeted therapies in autoimmune rheumatic disease: systematic literature review. Autoimmun Rev 18(5):535-541

Publisher's Note Springer Nature remains neutral with regard to jurisdictional claims in published maps and institutional affiliations. 